\title{
Risk factors for mortality among drug- resistant tuberculosis patients registered for drug-resistant treatment in Amhara region, Ethiopia: a historical cohort study
}

Daniel Bekele Ketema ${ }^{1 *}$ (D), Muluneh Alene ${ }^{1}$, Moges Agazhe Assemie ${ }^{1}$, Leltework Yismaw ${ }^{1}$ and Mehari Woldemariam Merid ${ }^{2}$

\begin{abstract}
Background: The emergency of drug resistant tuberculosis is a major public health concern worldwide including Ethiopia. However, little is known about risk factors of mortality among drug resistant tuberculosis patients in the study site. Thus, this study was aimed to estimate the risks factors for mortality among drug resistant tuberculosis patients registered for drug-resistant treatment in Amhara region, Ethiopia.

Methods: An institutional-based retrospective cohort study was carried out among laboratory-confirmed drugresistant tuberculosis patients $(n=498)$ who were initiated treatment for drug-resistant tuberculosis between September 1, 2010, and December 31, 2017. The demographic and clinical characteristics of the patients were obtained from the register of patients treated for drug-resistant tuberculosis. The data were entered using EpiData version 4.2 and exported to Stata Version 14.1 for further analysis. Descriptive measures were used to characterize the study participants. Kaplan-Meier was used to estimate the survival time of the patients. Cox proportional hazard model was used to identify risk factors for mortality. Hazard Ratio (HR) with 95\% confidence interval was used to report the strength of association between risk factors and mortality.

Results: Death was observed among 14.2\% $(n=70)$ of the 498 patients who met the inclusion criteria. The incidence rate of death was 8.20 (95\% Cl: 7.62, 20.50) per 1000 Person-months in the course of follow-up. The median age was 29.30 years (IQR:23-41). Age 45 years and above (adjusted hazard ratio (AHR) $=1.28: 95 \% \mathrm{Cl}: 1.10,1.68)$, smoking cigarette (AHR $=1.39: 95 \% \mathrm{Cl}: 1.27,3.18)$, tuberculosis related complication (AHR $=9.31: 95 \% \mathrm{Cl}: 5.11,16.97)$, anemia $(\mathrm{AHR}=$ 3.04:95\% Cl:1.14, 9.20), HIV/AIDS (AHR = 1.34:95\% Cl:1.25, 3.35), previous tuberculosis treatment history (AHR = 1.37:95\% Cl:1.16, 1.86), and diabetes mellitus (AHR $=1.85: 95 \%$ Cl:1.24,5.71) were identified risk factors for mortality.

(Continued on next page)
\end{abstract}

\footnotetext{
* Correspondence: dabekle121@gmail.com

'Department of Public Health, College of Health Science, Debre Markos University, Debre Markos, Ethiopia

Full list of author information is available at the end of the article
}

C C The Author(s). 2020 Open Access This article is licensed under a Creative Commons Attribution 4.0 International License, which permits use, sharing, adaptation, distribution and reproduction in any medium or format, as long as you give appropriate credit to the original author(s) and the source, provide a link to the Creative Commons licence, and indicate if changes were made. The images or other third party material in this article are included in the article's Creative Commons licence, unless indicated otherwise in a credit line to the material. If material is not included in the article's Creative Commons licence and your intended use is not permitted by statutory regulation or exceeds the permitted use, you will need to obtain permission directly from the copyright holder. To view a copy of this licence, visit http://creativecommons.org/licenses/by/4.0/ The Creative Commons Public Domain Dedication waiver (http://creativecommons.org/publicdomain/zero/1.0/) applies to the data made available in this article, unless otherwise stated in a credit line to the data. 


\begin{abstract}
(Continued from previous page)
Conclusions: This study concluded that drug-resistant tuberculosis mortality remains high in the study site. Age 45 years and above, smoking cigarette, tuberculosis related clinical complication, being anemic at baseline, HIV/AID, previous tuberculosis treatment history, and diabetes mellitus were identified risk factors for mortality. Continual support of the integration of TB/HIV service with emphasis and working on identified risk factors may help in reducing drug-resistant tuberculosis mortality.
\end{abstract}

Keywords: Risk factors, Drug-resistant tuberculosis, Mortality

\section{Background}

Tuberculosis (TB), a preventable and treatable disease, remains one of the important causes of death worldwide [1]. The World Health Organization (WHO), through the End TB Strategy [2], envisions the eradication of death, diseases, and suffering due to TB by 2035 [1]. However, Drug-Resistant TB (DR-TB) impede the realization of this vision [3] because of its lengthy, toxic, high cost of treatment, and poorer treatment outcome when compared to drug-susceptible TB. DR-TB is caused by TB bacteria that are resistant to at least one first-line anti-TB drug, while Multidrug-resistant TB (MDR-TB) is caused by TB bacteria that are resistant to at least isoniazid and rifampin, the 2 most potent TB drugs [1].

DR-TB remains major drug-resistant airborne infection and about one-third of deaths were attributed by DR-TB from antimicrobial resistance [4]. Overall, DR-TB causes about $10 \%$ of all TB deaths and it is a global threat $[5,6]$. According to WHO 2017, global TB report, 600,000 DR$\mathrm{TB}$ cases were estimated to be diagnosed globally and caused 240,000 deaths in 2016, and most deaths occurred in Asia and Africa region [7, 8]. Even though WHO has recommended shorter regimens (9-12-months) for specific groups of patients, majority DR-TB cases were treated for a minimum of 18-24 months with second-line TB drugs that have significant adverse effects [9].

A previous studies suggested that the prevalence of DRTB was progressively increased in sub-Saharan Africa, where health resources, finances, and the skilled personnel required for diagnosis and management are limited [10-12]. The global data shows that only $54 \%$ of MDR-TB patients are successfully treated due to high mortality and lost follow up [7].

Ethiopia is one of the 30 high burden DR-TB countries identified by WHO with an estimated 5.8/1000 new MDR/RR-TB cases per year in 2016 [1] . A previous studies done at St. Peter's specialised TB hospital in Addis Ababa, the capital city of Ethiopia, found that $15.30 \%$ known deaths with incidence rate of 3.6 per 10,000 person-days [13]. Other study conducted at University of Gondar specialized hospital demonstrated that 13.9\% DRTB patients were died and 5.9\% lost follow-up in the entire follow up time [14]. Therefore, understanding risk factors for DR-TB mortality is vital to improving DR-TB treatment outcomes. Previous studies have shown that increasing age $[15,16]$, smoking cigarette $[17,18]$, comorbidities [19-21] such as HIV/AIDS and diabetes, clinical complication $[14,21,22]$ therapeutic delay $[3,23,24]$ were contributing factors for DR-TB mortality .

Despite of the approval of standard TB prevention and control program, Ethiopia remains one of the high $\mathrm{TB}$ and DR-TB burden countries where TB remains a substantial cause of morbidity and mortality $[25,26]$. Knowledge on the risk of DR-TB mortality is critical for informing health policy solutions needed to improve the outcome of DR-TB care and contain the spread of disease. As mentioned above studies in other settings identified several factors for mortality of patients with DRTB patients. However, only few studies have evaluated the risk factors for mortality in Ethiopia [13, 14, 18], and none in Amhara region by including all the major three DR-TB treatment initiating center (Gondar university specialized comprehensive hospital, Debre Markos Referral Hospital and Boru Meda Hospital). Therefore, the aim of this study was to estimate the risk factors for mortality among drug-resistant tuberculosis patients registered for drug-resistant treatment in Amhara region, Ethiopia.

\section{Methods \\ Study design and settings}

An institutional based retrospective cohort study was conducted among DR-TB patients who have commencing treatment from September 1, 2010 to December 31, 2017 in Amhara region. Among DR-TB treatment initiating centers found in the region, University of Gondar Comprehensive Specialized Hospital, Boru-Meda Hospital, and Debre-Markos Referral Hospital were the three treatment centers which provides a services for more than $80 \%$ of the DR-TB patients found in the region. University of Gondar Comprehensive Specialized Hospital, which is located in North Gondar Administrative Zone, the Amhara Regional State, started DR-TB treatment as a pilot program with the Global Health Commute (GHC) to treat patients as a national response to the emerging threat of drug-resistant TB in September 2010. The second setting is Boru-Meda Hospital which located in South Wollo 
zone, Amhara regional sate. The third study site is setting is Debre-Markos Referral Hospital, which was found in Debre Markos town, Amhara region.

\section{Sample size determination}

All DR-TB patients who were enrolled at the University of Gondar Compressive Specialized Hospital, Debre Markos Referral Hospital and Boru-Meda Hospital from September 12,010 to December 31, 2017 were considered for this study. To check the efficiency, minimum adequacy of samples (427) was determined based on survival power formula as follow.

$$
\begin{gathered}
E=\frac{Z(a / 2)+Z \beta}{p 1(1-p 1)(\ln H R)^{2}} \\
N=\frac{E}{p(E)}
\end{gathered}
$$

Where: $E=$ require number of events, $\mathrm{p}(\mathrm{E})=$ is average probability of event among exposed individuals from previous study, $\mathrm{N}=$ minimal sample size, $\mathrm{p} 1=$ Proportion of subjects under exposure variable, and $\mathrm{HR}=$ hazard ratio.

\section{Assumption}

Two-sided $\mathrm{Z}$ value at $95 \%$ confidence interval $=1.96$, Power $=80 \%, \beta=0.2, Z \beta=0.842$.

Accordingly:

\begin{tabular}{llllll}
\hline Variables & HR & P1 & Event & Pro (event) & Sample size \\
\hline HIV co infection [14] & 2.6 & 0.217 & 53 & 0.23 & 230 \\
Anemia [27] & 2.2 & 0.37 & 56 & 0.2 & 281 \\
Drug susceptibility [18] & 2.33 & 0.21 & 66 & 0.178 & 371 \\
\hline
\end{tabular}

Accordingly, the maximum sample size based on the above formula after considering $15 \%$ incompleteness was 427. However, the actual data collected in a study area were 498 which maximize the true estimate of parameters.

\section{Study population}

We included all patients, who had laboratory confirmed DR-TB, and registered on DR-TB register book and started treatment between September 12,010 and December 31,2017.

\section{Exclusion criteria}

Patients who had not been assigned a treatment outcome at the end of the follow-up period, clinically diagnosed cases (non-laboratory confirmed cases) and transferred in cases from other health facilities were excluded from the analysis.

\section{Variables of the study}

Death, as defined by the national TB program, was the main outcome of this study. The Ethiopia TB programme, in accordance WHO classification, "death" as any patient who dies during the course of DR-TB treatment [7].

Independent variables considered in the analysis included (age, sex, anti-TB drug history, marital status, level of education, religion) and clinical (HIV status, diabetes mellitus, hypertension, asthma, types of resistant, initial smear and culture result) and behavioural (cigarette smoking).

\section{Definition of variables}

DR-TB treatment outcomes were assigned as per the definitions in the Ethiopian national TB guidelines, which have been adopted wholly from the WHO definitions and reporting framework for TB guidelines as cured, treatment completed, treatment failed, died, lost to follow-up and not evaluated [28].

\section{Cured}

patients completed treatment without evidence of treatment failure and had three or more consecutive negative culture taken 30 days apart.

\section{Treatment complete}

complete treatment without evidence of treatment failure but no recorded culture result.

\section{LTFU}

Patients who interrupt treatment 2 or more consecutive months.

Death

Referred to death for any reason during the course of treatment.

\section{Time to death}

Is the time gap, in months, between the beginning of 190 DR-TB treatment and the date of death.

\section{Censored}

If they had the TB treatment outcome of cured, completed, and transferred out or lost to follow-up or were still on treatment at the end of the study.

\section{Anemia}

Based on the WHO definition, patients were considered as anemic if their haemoglobin level $<12 \mathrm{~g} / \mathrm{dl}$ for female and children and less than $13 \mathrm{~g} / \mathrm{dl}$ for men.

\section{Source of data and procedure}

This study used secondary data that were collected using a structured data extraction checklist. Data were 
extracted from patients' DR-TB registration books, treatment follow-up sheet, green card and medical records. These data sources have contained a sociodemographic characteristic (age, sex, residence, marital status, educational status, occupation, religion), clinical variables (HIV status and other comorbidities, site of TB disease, number of previous TB treatments, initial DR-TB regimen, initial regimen change, initial sputum and culture result). Data were collected by workers who were working in the DR-TB treatment centre.

\section{Data quality management}

Training on the objective of the study and how to retrieve records as per data extraction sheet was given to data collectors and supervisors for 2 days before data collection started. The data extraction sheets were pretested for consistency of understanding of tools and completeness of data for charts. Necessary adjustment for the final data collection sheet was made.

\section{Data processing and analysis}

The data were checked for inconsistencies, coding error, completeness, accuracy, clarity, and missing values before they were entered. The data were entered using EpiData version 4.2 and exported to Stata Version 14.1 for further analysis. Descriptive measures were used to characterize the study participants. The time for death was estimated using the Kaplan-Meier method. The logrank test was used to compare hazard curves between baseline categorical variables. Incidence of death with

Table 1 Socio-demographic characteristics of drug-resistant tuberculosis patients in Amhara region, from September 1, 2010 to December 31, 2017

\begin{tabular}{lll}
\hline Variables & Frequency & Percent (\%) \\
\hline Sex & 261 & 52.4 \\
Male & 237 & 48.6 \\
$\quad$ Female & & \\
Marital status( $\boldsymbol{n}$ 494) & 248 & 50.2 \\
$\quad$ Never married & 183 & 37.1 \\
Married & 47 & 9.5 \\
Divorced & 16 & 3.2 \\
Widowed & & \\
Level of education & 222 & 44.6 \\
No education & 137 & 27.5 \\
Primary & 139 & 27.9 \\
Secondary and above & & \\
Residence & 238 & 47.8 \\
Urban & 260 & 52.2 \\
Rural & &
\end{tabular}

Table 2 Descriptive Statistics of continuous covariates of drugresistant tuberculosis patients in Amhara region, from September 1, 2010 to December 31, 2017

\begin{tabular}{ll}
\hline Variables & Median $[\mathrm{IQR}]$ \\
\hline Age $($ Year $)$ & $29.50[23,41]$ \\
$\mathrm{BMI}\left(\mathrm{Kg} / \mathrm{m}^{2}\right)$ & $17.51[15.82,21.00]$ \\
Hospital stay in day & $63[30 ., 90]$ \\
Number of previous treatment & $2[1,3]$ \\
Follow up time in month & $20[9,21]$ \\
\hline
\end{tabular}

BMI Body Mass Index, IQR Inter Quartile Range

respect to person time at risk was calculated. Variables which are significant at $P<0.20$ in the bivariable analysis were included in the final Cox- regression analysis. Proportionality assumption was tested by global test based on scheonfeld residuals. Hazard ratio and 95\% confidence interval were used to report the strength of association between mortality rate and it's risk factors.

Table 3 Baseline clinical characteristics of drug-resistant tuberculosis patients in Amhara region, from September 1, 2010 to December 31, 2017

\begin{tabular}{|c|c|c|}
\hline Variables & Frequency & Percent (\%) \\
\hline \multicolumn{3}{|c|}{ Baseline sputum smear } \\
\hline Positive & 395 & 79.3 \\
\hline Negative & 103 & 20.7 \\
\hline \multicolumn{3}{|c|}{ Body mass index } \\
\hline$<18.5$ & 383 & 76.9 \\
\hline$\geq 18.5$ & 115 & $23 . .1$ \\
\hline \multicolumn{3}{|l|}{ Diabetes } \\
\hline Yes & 16 & 3.2 \\
\hline No & 482 & 96.8 \\
\hline \multicolumn{3}{|l|}{ HIV/AIDS } \\
\hline Positive & 123 & 24.7 \\
\hline Negative & 375 & 75.3 \\
\hline \multicolumn{3}{|l|}{ Anaemia } \\
\hline Anemic & 387 & 77.7 \\
\hline Non anemic & 111 & 22.3 \\
\hline \multicolumn{3}{|c|}{ Previous tuberculosis treatment history } \\
\hline Yes & 399 & 80.1 \\
\hline No & 99 & 19.9 \\
\hline \multicolumn{3}{|c|}{ Tuberculosis type } \\
\hline Pulmonary & 442 & 88.7 \\
\hline Disseminate & 56 & 11.3 \\
\hline \multicolumn{3}{|c|}{ Smoking cigarette } \\
\hline Yes & 72 & 14.5 \\
\hline No & 426 & 85.5 \\
\hline
\end{tabular}



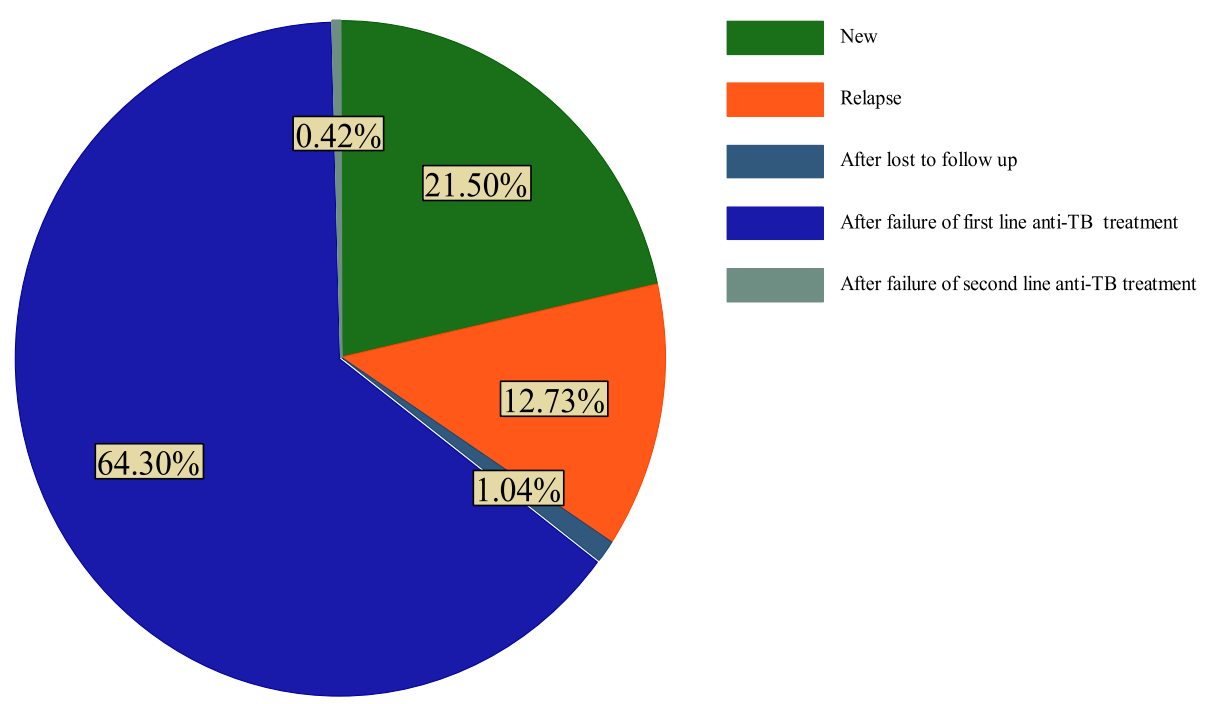

Fig. 1 Registration group of drug-resistant tuberculosis patients in Amhara region, from September 1, 2010 to -December 31, 2017

\section{Results}

Baseline demographic and clinical characteristics

A total of 565 patients were registered for DR-TB treatment between September 1, 2010 and December 31, 2017. We analysed data from 498 laboratory confirmed DR-TB patients, after excluding patients who were clinically diagnosed $(n=15)$, transferred in from other health facility $(n=35)$ and patients who had not been assigned a treatment outcome at the end of the followup period $(n=17)$. Among a total of 498 study participants, almost half 261 (52.4\%) were male (Table 1).

The median age of DR-TB patients was 29.30 years [IQR:23-41 years]. The length of hospital stay was higher for patients who were died compared to patients who did not with median of 63 days and 54 days respectively. The median body mass index (BMI) of DR-TB patients at baseline was $17.51 \mathrm{Kg} / \mathrm{m}^{2}$ [IQR: 15.82-21.00] (Table 2).

About 421 (84.5\%) patients had pulmonary TB, of which 79.3 and $96.4 \%$ patients had positive sputum smear and positive culture result at baseline respectively. With regard to drug-resistant patterns, half $(50.4 \%)$ of patients were mono resistant, $41.2 \%$ were multidrugresistant, $8.2 \%$ poly-resistant, and $0.2 \%$ extensive drugresistant (Table 3). Among 498 DR-TB patients, nearly two third $320(64 \%)$ of patients were enrolled after failure of first line anti-TB treatment followed by newly diagnosed cases 107 (21.5\%) (Fig. 1).

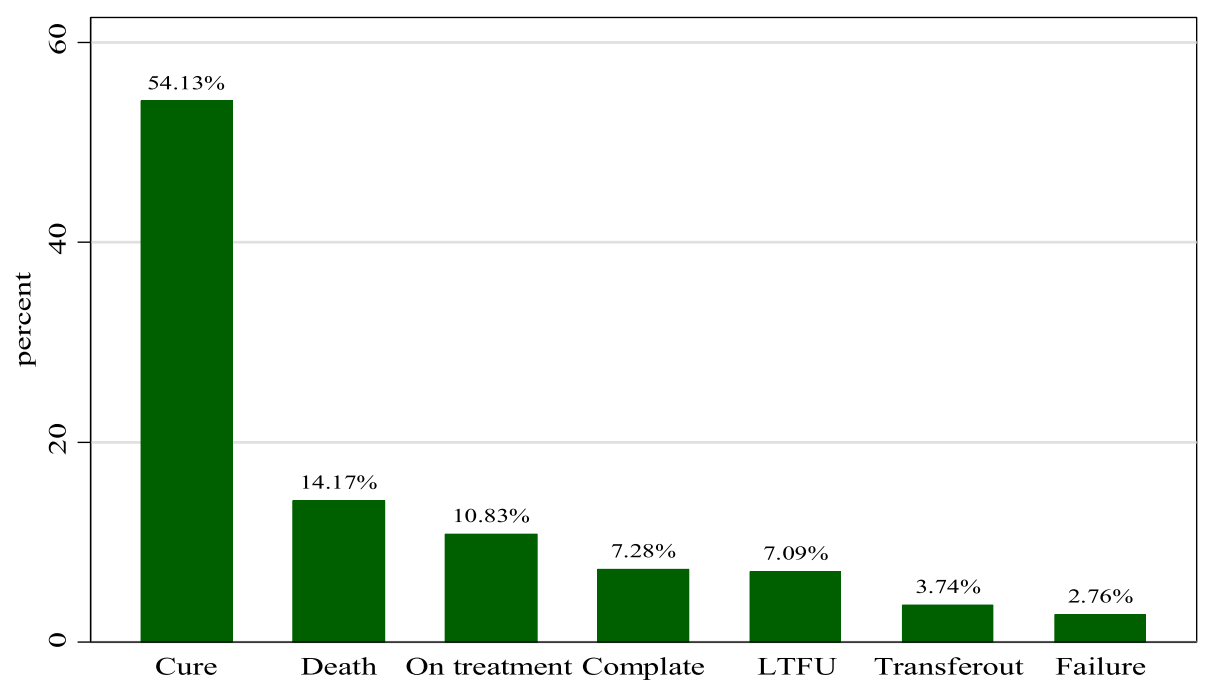

Fig. 2 Treatment outcome of drug-resistant tuberculous patients in Amhara region, from September 1, 2010 to December 31, 2017 


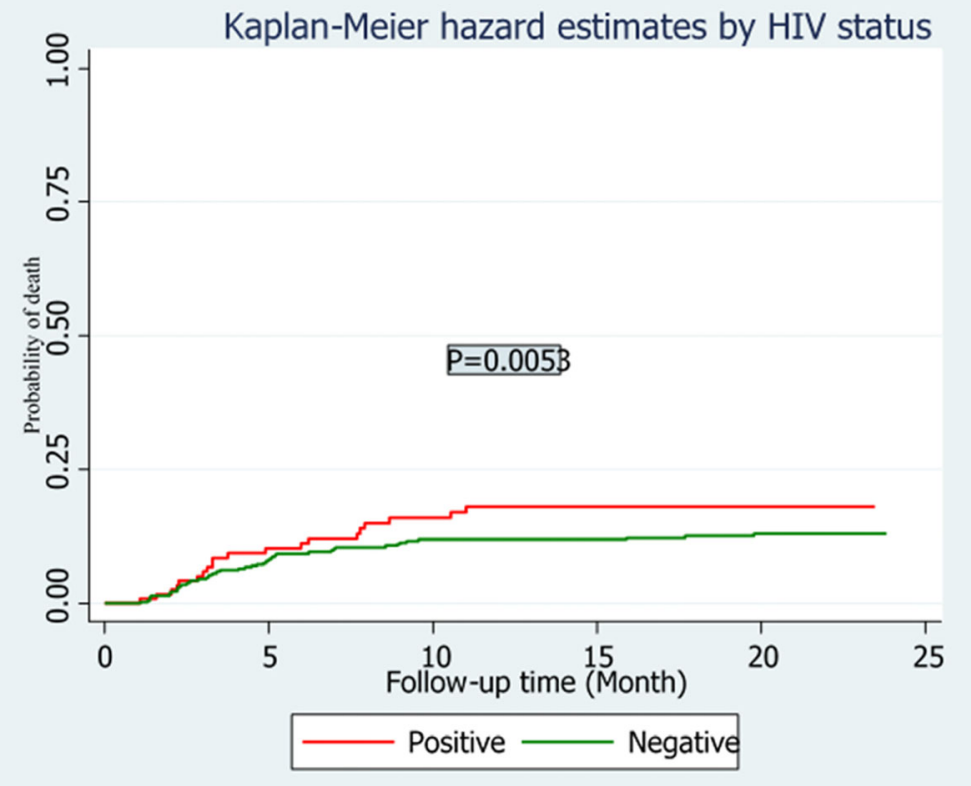

Fig. 3 Kaplan-Meier hazard curve and Log Rank test by HIV status for drug-resistant tuberculosis patients in Amhara region from September 1, 2010 to December 31, 2017

\section{Treatment outcomes and survival status}

The overall treatment success rate of 498 study participants was $61.4 \%$ (cure $54.1 \%$ and treatment completion $7.3 \%$ ). In addition, in the follow up time,70 (14.1\%) died, 14 (2.8\%) had treatment failure,36 (7.1\%) were lost to follow-up in the course of treatment (Fig. 2). The overall incidence density of death in the cohort was 8.20 (95\% CI: 7.6, 20.5) per
1000 Person-months. Higher mortality rate was observed among individuals with HIV/AIDS with incidence rate of 7.4 per 1000 person-months compared to 1.1 per 1000 person-months among HIV negative patients. The log rank test also showed that the hazard rate of mortality was significantly higher among HIV positive patients as compared to HIV negative patients (Fig. 3). Based on the patient's

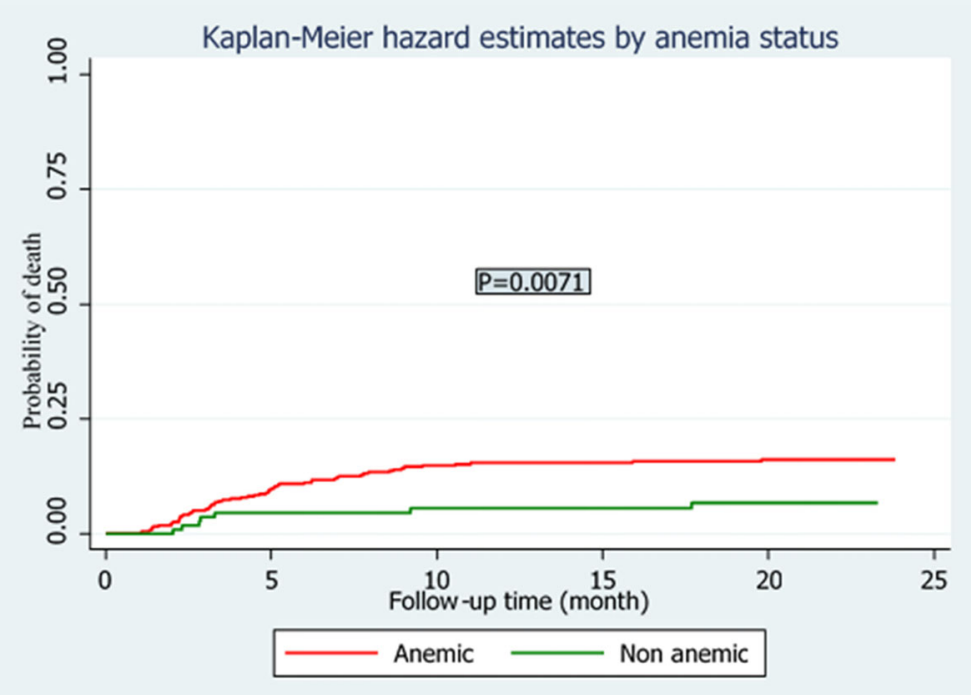

Fig. 4 Kaplan-Meier hazard curve and Log Rank test by the level of haemoglobin (anemic vs no anemic) of drug-resistant tuberculosis patients in Amhara region from September 1, 2010 to December 31, 2017 
level of haemoglobin (categorized as Anemic and non anemic), the hazard of mortality was worsened for anemic patients as compared to non anemic patients in the course of follow-up time (Fig. 4).

\section{Predictors of DR-TB mortality}

In the multivariate cox proportional hazard model, Age 45 years and above, smoking cigarette, tuberculosis related clinical complication, being anemic at baseline, HIV/AID, previous tuberculosis treatment history, and diabetes mellitus were significant risk factors for mortality (Table 4).

\section{Discussions}

The objective of this study was to estimate the risk factors for mortality among DR-TB patients in Amhara

Table 4 Multivariable Cox regression analysis for risk factors for mortality among drug-resistant tuberculosis patients in Amhara region, from September 1, 2010 to December 31, 2017

\begin{tabular}{lll}
\hline Variables & $\mathrm{CHR}[95 \% \mathrm{Cl}]$ & AHR $[95 \% \mathrm{Cl}]$ \\
\hline Age in years & & \\
$<=24$ & 1.00 & 1.00 \\
$25-44$ & $0.42\left[\begin{array}{lll}0.21 & 0.88\end{array}\right]$ & $0.45[0.141 .84]$ \\
45 and above & $1.18\left[\begin{array}{lll}1.12 & 1.43\end{array}\right]$ & $1.28\left[\begin{array}{lll}1.10 & 1.68\end{array}\right]$
\end{tabular}

Tuberculosis related complication

$\begin{array}{lll}\text { No } & 1.00 & 1.00 \\ \text { Yes } & 11.94[7.14 \text { 19.97] } & 9.31[5.1116 .97]\end{array}$

Baseline sputum smear

$\begin{array}{lll}\text { Positive } & 1.00 & 1.00 \\ \text { Negative } & 1.56\left[\begin{array}{lll}0.75 & 3.27\end{array}\right] & 1.23[0.652 .33]\end{array}$

Level of education

No education

2.69 [1.34 5.39] $\quad 1.29$ [0.62 2.69]

Primary

$1.67[0.753 .68]$

Secondary and above

1.00

$1.66[0.743 .70]$

Anaemia

Non anaemic

1.00

1.00

Anaemic

[1.35 8.41]

$3.04[1.149 .20]$

\section{HIV/AIDS}

Negative

$1.00 \quad 1.00$

Positive

$1.42[1.214 .84]$

1.34 [1.25 3.35]

Diabetes mellitus

$\begin{array}{lll}\text { No } & 1.00 & 1.00 \\ \text { Yes } & 2.52[2.147 .31 .] & 1.85[1.245 .71]\end{array}$

\section{Previous tuberculosis treatment history}

\begin{tabular}{lll} 
No previous treatment history & 1.00 & 1.00 \\
Had previous treatment history & $1.57\left[\begin{array}{ll}1.22 & 1.79]\end{array}\right.$ & $1.37[1.161 .86]$ \\
Smoking cigarette & & \\
No & 1.00 & 1.00 \\
Yes & $1.89\left[\begin{array}{ll}1.04 & 3.36\end{array}\right]$ & $1.39[1.273 .18]$ \\
\hline
\end{tabular}

region: Ethiopia. This study found that incidence of mortality was 8.2 per 1000 person-months observation. This finding was comparable with previous studies conducted in Ethiopia [14, 18], and other countries in subSaharan Africa [23]. In contrary, our finding was higher compared to the research conducted in St. Peter specialized TB hospital in Addis Ababa which is 3.6 per 10,000 person-days [13]. This difference may be the previous study only include DR-TB cases enrolled in one DR-TB treatment initiating center and only 188 DR-TB cases were included in the analysis.

Our study determined that being aged 45 years and above was a significant risk factor for mortality. This finding was consistent with findings reported in Eastern Cape Province, South Africa [29]. This evidence was supported by previously existing literature [30] which indicated that increased age is associated with increasing co-morbidities as well as weakening of body functions. As age increased the prevalence of comorbidities such as diabetes were also increased and parallel the risk of DRTB mortality increased.

The study found that smoking cigarette was associated with mortality. This finding was in agreement with other studies elsewhere [17, 18]. Probably cigarette smoking speculated to lower cytokine-producing macrophages with diminished influx of interferon gamma producing effector T-cells in the lungs, which leads to increase the incidence of active and latent pulmonary $\mathrm{TB}$ as well poor clinical prognosis.

The study determined that HIV co-infection was a risk factor for mortality. This finding is consistent with previous studies conducted in Ethiopia [13, 18, 23]. This is due to HIV co-infection reduced the integrity and function of CD4+ cells which reduced the level of immunity and increased risk of mortality [31, 32] In addition, TB hand increases HIV replication and viral diversification rates, by increasing pro inflammatory cytokine production, which increase HIV viral replication and diversity, which in turn facilitating immune escape [33, 34]. This synergistic effect may be the principal reason for the high mortality among HIV confected DR-TB patients.

In this study patients who had a tuberculosis-related clinical complications have high risk of mortality. The presence of clinical complication were the sign of poor progression of diseases and subsequent increase in the risks of mortality. This finding was in consistent with studies conducted in Ethiopia [13, 14, 18].

This study also demonstrate that anemia considerably increase the risk of mortality when compared to nonanemic patients. This might be related with, anemic patients may liable to adverse drug effect during antituberculosis treatment. This finding was comparable with other study which found that anaemia is associated with drug-resistant tuberculosis mortality [35]. In 
addition, anemic patients might have increased risks of infection and have compromised immunity which contribute for advancement of disease progression and risk of death.

Drug-resistant tuberculosis patients with diabetes mellitus co-morbidity have increased risk for mortality. This association has been demonstrated in the previous studies [20, 21, 36]. A possible justification might be, patients with diabetes mellitus have impaired immunity compared to healthy individuals, and sequel of diabetes may potentiate the adverse effects of anti-tuberculosis drugs.

\section{Limitation of the study}

There was a limitation for this study. Since the study was based on secondary data; potential important variables such as radiograph findings and behavioural factors were not assessed to define the best predictors of mortality. Other limitation of this study was nontuberculosis related death such as accidents or other chronic diseases my cause death in the course of treatment, but specific cause of death was not available. This may lead to over estimation of the incidence of death rate in our study. Therefore, is recommended to computing risk analysis for each specific cause of death in the course of treatment.

\section{Conclusions}

This study concluded that drug-resistant tuberculosis mortality remains high in the study site. Age 45 years and above, smoking cigarette, tuberculosis related clinical complication, being anemic at baseline, HIV/AID, previous tuberculosis treatment history, and diabetes mellitus were identified risk factors for mortality. Continual support of the integration of TB/HIV service with emphasis and working on identified risk factors may help in reducing drug-resistant tuberculosis mortality.

\section{Abbreviations}

DR-TB: Drug Resistant Tuberculosis; MDR-TB: Multidrug Resistant

Tuberculosis,; HIV: Human immune Deficiency Virus; WHO: World Health Organization; DOTS: Direct observed treatment, short course; DM: Diabetes Mellitus; AIC: Akaike information criteria

\section{Acknowledgements}

The author would like to acknowledge, University of Gondar's Comprehensive Specialized Hospital, Debre-Markos Referral Hospital and Boru-Meda Hospital for making the data for this research undertaking available. The authors are also grateful to data collectors and supervisors.

\section{Authors' contributions}

DBK originated the research idea and analyzed the data. MA, MAA, LYW, and $\mathrm{MWH}$ contributed to data analysis, writing and substantively revised the manuscript. All authors have read and approved the manuscript, and ensure that this is the case.

\section{Funding}

No funding available for this study.
Availability of data and materials

The datasets used and/or analysed during the current study are available from the corresponding author on reasonable request.

\section{Ethics approval and consent to participate}

Ethical approval was obtained from the ethical review committee of Institute of Public Health, University of Gondar. Letter of cooperation for all selected Hospitals was obtained from Amhara National Health Bureau, Amhara Public Health Institute. Informed consent had not been required in our case as whole data had been retrieved from the medical records of patients who had already completed treatment or had some labeled treatment outcome. Data were anonyms and held on a secure password protected system.

Confidentiality during all phases of research activities was kept.

\section{Consent for publication}

Not applicable.

\section{Competing interests}

The authors declare that they have no competing interests.

\section{Author details}

'Department of Public Health, College of Health Science, Debre Markos University, Debre Markos, Ethiopia. ${ }^{2}$ Department of Epidemiology and Biostatistics, Institute of Public Health, College of Medicine and Health Sciences and Specialized Comprehensive Hospital, University of Gondar, Gondar, Ethiopia.

Received: 23 April 2020 Accepted: 8 July 2020

Published online: 31 July 2020

\section{References}

1. World Health Organization, Global tuberculosis report 2017: World Health Organization; 2017. Report No: 9241565055, 2018.

2. Uplekar M, et al. WHO's new end TB strategy. Lancet. 2015;385(9979):1799-801.

3. Narasimooloo R, Ross A. Delay in commencing treatment for MDR TB at a specialised TB treatment centre in KwaZulu-Natal. S Afr Med J. 2012;102(6): 360-1.

4. Global TB Caucus. Price of a pandemic 2017. 2017. 2017 [cited 2020 13]; Available from: https://www.globaltbcaucus.org/en-research.

5. All party Parliamentary group on global Tuberculosis, Drug-resistant tuberculosis old disease New threat, summary version. 2013 [cited 32 13]; Available from: https://www.appg-tb.org.uk/publications.

6. Fantaw D, et al. Assessment of the survival status and risk factors for the mortality among multidrug resistant tuberculosis patients at Adama and Bishoftu general hospitals, Oromia, Ethiopia: a retrospective cohort study. Adv Pharmacoepidemiol Drug Saf. 2018;7(220):2167-1052.1000222.

7. WHO. Global tuberculosis report 2017. Geneva: World Health Organization; 2017.

8. Organization, W.H. Global tuberculosis control: surveillance, planning, financing: WHO report 2008, vol. 393: World Health Organization; 2008.

9. Mukherjee JS, et al. Programmes and principles in treatment of multidrugresistant tuberculosis. Lancet. 2004;363(9407):474-81.

10. Amor $\mathrm{YB}$, et al. Underreported threat of multidrug-resistant tuberculosis in Africa. Emerg Infect Dis. 2008;14(9):1345.

11. Buthelezi S. Situational analysis of TB drug resistance in KwaZulu-Natal province: Republic of South Africa. in 2nd Meeting of the Global XDR TB Task Force; 2008.

12. Chirenda, J., et al. The trend of resistance to anti-tuberculosis drugs in Botswana: results from the 4th national anti-tuberculosis drug resistance survey. in 40th Union World Conference on Lung Health. 2009.

13. Getachew T, Bayray A, Weldearegay B. Survival and predictors of mortality among patients under multi-drug resistant tuberculosis treatment in ethiopia: st. peter's specialized tuberculosis hospital, ethiopia. Int J Pharm Sci Res. 2013:4(2):776.

14. Meressa D, Hurtado RM, Andrews JR, Diro E, Abato K, Daniel T, Prasad P, Prasad R, Fekade B, Tedla Y. Achieving high treatment success for multidrug-resistant TB in Africa: initiation and scale-up of MDR TB care in Ethiopia-an observational cohort study. Thorax. 2015;70(12):1181-8 thoraxjnl-2015-207374.

15. Chiang $C-Y$, et al. Outcome of pulmonary multidrug-resistant tuberculosis: a 6-yr follow-up study. Eur Respir J. 2006;28(5):980-5. 
16. Abuaku B, et al. Treatment default and death among tuberculosis patients in Hunan, China. Scand J Infect Dis. 2010;42(4):281-7.

17. Wang J, Shen H. Review of cigarette smoking and tuberculosis in China: intervention is needed for smoking cessation among tuberculosis patients. BMC Public Health. 2009;9(1):292.

18. Molalign $\mathrm{S}$, Wencheko E. Risk factors of mortality in patients with multi-drug resistant TB. Ethiop J Health Dev. 2015;29(2):83-8.

19. Kvasnovsky $C L$, et al. Extensively drug-resistant $T B$ in eastern cape, South Africa: high mortality in HIV-negative and HIV-positive patients. J Acquir Immune Defic Syndr. 2011;57(2):146-52.

20. Chung-Delgado K, et al. Mortality among MDR-TB cases: comparison with drug-susceptible tuberculosis and associated factors. PLoS One. 2015;10(3): e0119332.

21. Workneh MH, Bjune GA, Yimer SA. Diabetes mellitus is associated with increased mortality during tuberculosis treatment: a prospective cohort study among tuberculosis patients in south-eastern Amahra region, Ethiopia. Infect Dis Poverty. 2016;5(1):22.

22. Alene KA, et al. Treatment outcomes in patients with multidrug-resistant tuberculosis in north-West Ethiopia. Tropical Med Int Health. 2017;22(3):351-62.

23. Mollel EW, Chilongola JO. Predictors for mortality among multidrug-resistant tuberculosis patients in Tanzania. J Trop Med. 2017;2017:9241238.

24. Ahmadi F, et al. Epidemiologic survey of extra-pulmonary tuberculosis in Ahvaz from 2008 to 2013. Clin Epidemiol Global Health. 2020:802-5.

25. Reves R, Angelo S. As Ethiopia moves toward tuberculosis elimination, success requires higher investment. Washington DC: Center for Strategic and International Studies; 2016

26. Kebede $\mathrm{AH}$, et al. The first population-based national tuberculosis prevalence survey in Ethiopia, 2010-2011. Int J Tuberc Lung Dis. 2014;18(6): 635-9.

27. Alene Kefyalew Addis KV, McBryde ES, Emma S, Tsegaye AT, Archie CA. Clements, Treatment outcomes in patients with multidrug-resistant tuberculosis in north-west Ethiopia. Tropical Med Int Health. 2017;22(3):351-62.

28. Biruck Kebede B, et al. Guidelines on Programmatic Management of Drug Resistant Tuberculosis in Ethiopia. 2nd ed. Addis Ababa: Health FDROEMO; 2013.

29. Chingonzoh R, et al. Risk factors for mortality among adults registered on the routine drug resistant tuberculosis reporting database in the Eastern Cape Province, South Africa, 2011 to 2013. PLoS One. 2018;13(8):e0202469.

30. Falzon D, et al. Exploring the determinants of treatment success for tuberculosis cases in Europe. Int J Tuberc Lung Dis. 2005;9(11):1224-9.

31. Gupta A, et al. Tuberculosis incidence rates during 8 years of follow-up of an antiretroviral treatment cohort in South Africa: comparison with rates in the community. PLoS One. 2012;7(3):e34156.

32. Sonnenberg $P$, et al. HIV-1 and recurrence, relapse, and reinfection of tuberculosis after cure: a cohort study in south African mineworkers. Lancet. 2001;358(9294):1687-93.

33. Kalsdorf $\mathrm{B}$, et al. Relationship between chemokine receptor expression, chemokine levels and HIV-1 replication in the lungs of persons exposed to M ycobacterium tuberculosis. Eur J Immunol. 2013:43(2):540-9.

34. Juffermans NP, et al. Patients with active tuberculosis have increased expression of HIV coreceptors CXCR4 and CCR5 on CD4+ T cells. Clin Infect Dis. 2001;32(4):650-2.

35. Alene KA, et al. Treatment outcomes of patients with multidrug-resistant and extensively drug resistant tuberculosis in Hunan Province, China. BMC Infect Dis. 2017;17(1):573.

36. Tang S, et al. Risk factors for poor treatment outcomes in patients with MDR-TB and XDR-TB in China: retrospective multi-center investigation. PLoS One. 2013;8(12):e82943.

\section{Publisher's Note}

Springer Nature remains neutral with regard to jurisdictional claims in published maps and institutional affiliations.

Ready to submit your research? Choose BMC and benefit from:

- fast, convenient online submission

- thorough peer review by experienced researchers in your field

- rapid publication on acceptance

- support for research data, including large and complex data types

- gold Open Access which fosters wider collaboration and increased citations

- maximum visibility for your research: over $100 \mathrm{M}$ website views per year

At BMC, research is always in progress.

Learn more biomedcentral.com/submissions 